\title{
Heritability of the spatial distribution and peak density of macular pigment: a classical twin study
}

RE Hogg 1,2 , EL Ong 2,3, M Chamberlain², M Dirani², PN Baird ${ }^{2}$, RH Guymer ${ }^{2,4}$ and F Fitzke ${ }^{3,4}$

\begin{abstract}
Purpose To elucidate the heritability of peak density and spatial width of macular pigment (MP) using a Classical Twin Study. Methods Fundus autofluorescence images were obtained at $488 \mathrm{~nm}$ from 86 subjects or 43 twin pairs (21 monozygotic (MZ) and 22 dizygotic (DZ)) (27 male, 59 female) aged from 55 to 76 years (mean $62.2 \pm 5.3$ years). The relative topographic distribution of MP was measured using a grey scale of intensity (0-255 units) in a $7^{\circ}$ eccentricity around the fovea. Relative peak MP density (rPMPD) and relative spatial distribution of MP (rSDMP) were used as the main outcome measure in the statistical analysis.
\end{abstract}

Results A significantly higher correlation was found within MZ pairs as compared with that within DZ pairs for rPMPD, $(r=0.99,95 \%$ confidence interval $(95 \% \mathrm{CI}) 0.93$ to 1.00$)$ and $0.22,95 \% \mathrm{CI}-0.34$ to 0.71 ), respectively, suggesting strong heritability of this trait. When rSDMP was compared, there was no significant difference between the correlations within $\mathrm{MZ}$ pairs $(r=0.48,95 \% \mathrm{CI}-0.02$ to 0.83$)$ and $\mathrm{DZ}$ pairs ( $r=0.63,95 \%$ CI 0.32 to 0.83 ), thus rSDMP is unlikely to have a considerable heritable component. In addition, there was no difference between any MP parameter when normal maculae were compared with early age-related macular degeneration (AMD) (rPMPD 0.36 vs $0.34, t=1.18 P=0.243$, rSDMP 1.75 vs 1.75 , $t=0.028 P=0.977$ ).

Conclusions rPMPD is a strongly heritable trait whereas rSDMP has minimal genetic influence and a greater influence by environmental factors. The presence of macular changes associated with early AMD did not appear to influence any of these pigment parameters.

Eye (2012) 26, 1217-1225; doi:10.1038/eye.2012.98; published online 29 June 2012
Keywords: twin study; macular pigment; lutein; zeaxanthin; heritability

\section{Introduction}

Macular Pigment (MP) is composed principally of the polar carotenoids lutein $(\mathrm{L})$ and zeaxanthin (Z). Despite the fact that there are up to 50 dietary carotenoids, and approximately 14 carotenoids in human serum, ${ }^{1}$ only $\mathrm{L}$ and $\mathrm{Z}$ are selectively concentrated within the retina. ${ }^{2}$

Much interest has focused on MP owing to its location and inherent properties in that it is concentrated in the area of the retina that is most susceptible to age-related degenerative change and it can act as both a chain-breaking antioxidant and short-wavelength light filter. ${ }^{3-5}$ Those with a family history of advanced agerelated macular degeneration (AMD) have been shown to have lower MP, whereas some studies have shown a difference in MP between those with early AMD (drusen $\geq 63 \mu$ m or pigmentary irregularities also referred to as age-related maculopathy) and those without, ${ }^{6-8}$ although these relationships have not been consistent. ${ }^{9-11}$ Therefore, it has been postulated that MP may protect the retina from oxidative stress $6,7,12$ and age-related neuronal loss, thereby reducing the risk of AMD.

Large interindividual differences in MP have been demonstrated with several large populations-based studies showing that peak MP density (PMPD) can vary by over a factor of 10 between individuals. ${ }^{13,14}$ Subsequent studies have suggested a number of parameters such as age, diet, percentage body fat, gender and tobacco use ${ }^{15-19}$ might be determinants of MP; however, these only account for approximately a third of the variance leaving a significant proportion unexplained. In addition, despite
${ }^{1}$ Centre for Vision and Vascular Science, Queen's University, Belfast, UK

${ }^{2}$ Centre for Eye Research Australia, University of Melbourne, Royal Victorian Eye and Ear Hospital, Melbourne, Victoria, Australia

${ }^{3}$ Department of Visual Neuroscience, UCL Institute of Ophthalmology, London, UK

Correspondence: RE Hogg, Centre for Vision and Vascular Science, Institute of clinical science A, Queen's University, Grosvenor Road, Belfast BT12 6BA, UK Tel: +44 (0)289063 5018; Fax: +44 (0)289063 2699. E-mail: r.e.hogg@qub.ac.uk ${ }^{4}$ Joint last authors.

Received: 8 November 2011 Accepted in revised form: 2 February 2012 Published online: 29 June 2012 
MP's dietary origin only modest correlations exist with serum and dietary levels of $\mathrm{L}$ and $\mathrm{Z}$. Although the level of MP can be augmented in most people by diet or supplementation, the response is variable and not always correlated with baseline level, with most

supplementation studies identifying a subgroup of 'nonresponders' ${ }^{20}$ Given the possibility of idiosyncratic differences in absorption, transport, and retinal capture of dietary carotenoids, Liew et $a l^{21}$ examined the determinants of MP in a Classic Twin Study and concluded that genetic background is an important determinant of MP optical density. Liew et $a l^{21}$ only analysed PMPD, despite imaging techniques such as fundus autofluorescence (FAF) imaging allowing the spatial distribution of MP density (SDMP) to be identified and this has been shown to also vary significantly within individuals. ${ }^{22,23}$ In general, the MP concentration peaks at the foveal centre where $\mathrm{Z}$ predominates and declines rapidly with eccentricity into the parafovea where $\mathrm{L}$ becomes the dominant carotenoid. ${ }^{24-26}$ However, imaging techniques have revealed interesting variations in SDMP such as varying peak widths and ring-like structures, ${ }^{23,27,28}$ anatomical differences such as foveal width and slope ${ }^{29,30}$ can partially account for these although the reasons for their existence are not fully known.

We therefore explored both PMPD and SDMP assessed from FAF images in a group of older twins who were enrolled as part of the Melbourne AMD Twin Study in order to investigate the relative contribution of genetic and environmental factors that may determine the variation in these parameters in older subjects without advanced AMD.

\section{Materials and methods}

\section{A classical twin study}

A classical twin model compares the phenotypic resemblance between monozygotic (MZ) twin pairs who share all of their genotype with dizygotic (DZ) twin pairs who share up to $50 \%$ of their genes while assuming a shared environment within pairs irrespective of zygosity. The premise of such studies is that greater similarity will be found within $\mathrm{MZ}$ compared with $\mathrm{DZ}$ twin pairs if the trait has a significant heritable component.

\section{Twin recruitment}

Participants (MZ and DZ twin pairs) were enrolled through the National Health and Medical Research Council-funded Australian Twin Registry (ATR) as part of the AMD twin study carried out by the Centre for Eye Research Australia, University of Melbourne. All twins over the age of 50 on the register were invited to attend.
Detailed inclusion and exclusion has been outlined elsewhere. ${ }^{31,32}$ Within the AMD twin study each twin underwent a standard questionnaire, a comprehensive eye examination and intraocular photography according to protocols published elsewhere. ${ }^{31,32}$

\section{Statement of ethics}

The study was developed in accordance with the tenets of the Declaration of Helsinki and ethics approval for the study was obtained from both the Royal Victorian Eye and Ear Hospital Human Research and Ethics Committee and the ATR. Each subject provided written informed consent before participation.

\section{Zygosity}

Twin zygosity was determined by asking a standardized and validated collection of questions used by the ATR. ${ }^{33}$ Further details have been outlined elsewhere. ${ }^{31,32}$

\section{Image acquisition}

FAF imaging was undertaken using a cSLO (Heidelberg Retinal Angiograph 2 (HRA-2), Heidelberg Engineering, $\mathrm{GmbH}$, Dossenheim, Germany). A solid-state laser $(488 \mathrm{~nm})$ was used to illuminate the fundus and the induced fluorescence was recorded through a long-pass filter with a short-wavelength cutoff at $500 \mathrm{~nm}$. A twowavelength method is the more commonly accepted method for MP assessment using FAF owing to concerns about the comparison of absolute values with the onewavelength method and its ability to deal with nonuniform fluorophore distribution. ${ }^{34}$ However, in this study we are not comparing absolute values as would be the case in a population-based study, we are comparing the similarity between pairs of individuals. This study is, therefore, less susceptible to the drawbacks of the methodology.

FAF images were only obtained from those twins examined in clinics where a HRA-2 was available. A retinal area $30^{\circ} \times 30^{\circ}$ centred on the fovea was recorded digitally at $256 \times 256$ pixel $(5 \mu \mathrm{m} /$ pixel $)$ and 256 -level grey-scale resolution. The images obtained were anonymized and analysed at the Institute of Ophthalmology in London. The anonymization was such that it prevented the grader from knowing which subjects were pairs.

\section{Image preprocessing and analysis}

Image sets were graded manually on a scale: 1 (worst quality) to 5 (best quality) by comparing with a set of standards, those graded 3 and above were included in the analysis. 
All images were aligned according to structural landmarks (ie, retinal blood vessels for images of the same eye, and the optic disc head for images of different eyes) using Picture Window Pro 4.0 (Digital Light \& Colour, Belmont, MA, USA) to ensure that the images being compared were as closely aligned as possible. The images were then cropped to a standard size of $420 \times 420$ pixels to account for the differences in alignment.

All the images were adjusted for brightness, contrast and noise using a customized programme 'enhance_contrast.m' (MatLab 7.0.1, MathWorks Inc., Natick, MA, USA). Contrast was enhanced using histogram stretching whereby the range of intensity values is stretched so that it spans the full range from $0-255$. Taking $7 \times 7$ pixel boxes and averaging the number of pixels in each box removed noise.

\section{Calculation of the SDMP using autofluorescence imaging}

The FAF images were obtained using a HRA-2 cSLO that illuminated the fundus with an argon laser radiation at $488 \mathrm{~nm}$. As MP is located in the nerve fibre layer of the retina, it blocks the fluorescence coming from the underlying RPE so that the intensity of the $488 \mathrm{~nm}$ laser exciting radiation is reduced in regions of the retina where MP is located. These areas appear dark in the image. In order to analyse the topographic distribution of MP in FAF images, a computer programme (MatLab 7.0.1, MathWorks Inc.) was designed to carry out the following process: the topographic distribution of FAF across the retina was measured using a grey-scale index of intensity (0-255 units) along the horizontal and vertical meridians, intersecting at the foveal pixel. (Figure 1) This required a single observer to select the centre of the fovea, after which a $7^{\circ}$ radius circle was drawn around the foveal centre and mean values were calculated out to $7^{\circ}$ in $20 \times 20$ pixel blocks along the horizontal and vertical profiles. This eccentricity was chosen to encompass the area within which measurable MP has been shown to occur (Bone et al ${ }^{24}$ demonstrated that at $7^{\circ}$ the level drops to $3 \%$ of peak height and concluded that beyond $7^{\circ}$ the level could be practically considered as zero).

Relative optical density was calculated as $\log \left(R_{7} / R\right)$, where $R_{7}$ is the radiance equivalent of the mean greyscale value at $7^{\circ}$ and $R$ is the radiance equivalent value at any eccentricity. $R_{7}$ was calculated from the mean of 1016 pixels, arranged in a 1-pixel wide $7^{\circ}$ circle centred on the fovea. This functioned to reduce the effect of artifacts due to retinal blood vessels, which in some subjects encroached on the pigmented area of the macula, producing a negative optical density value. Optical density values were also scaled by a factor of 1.28 in order to compensate for the difference between MP

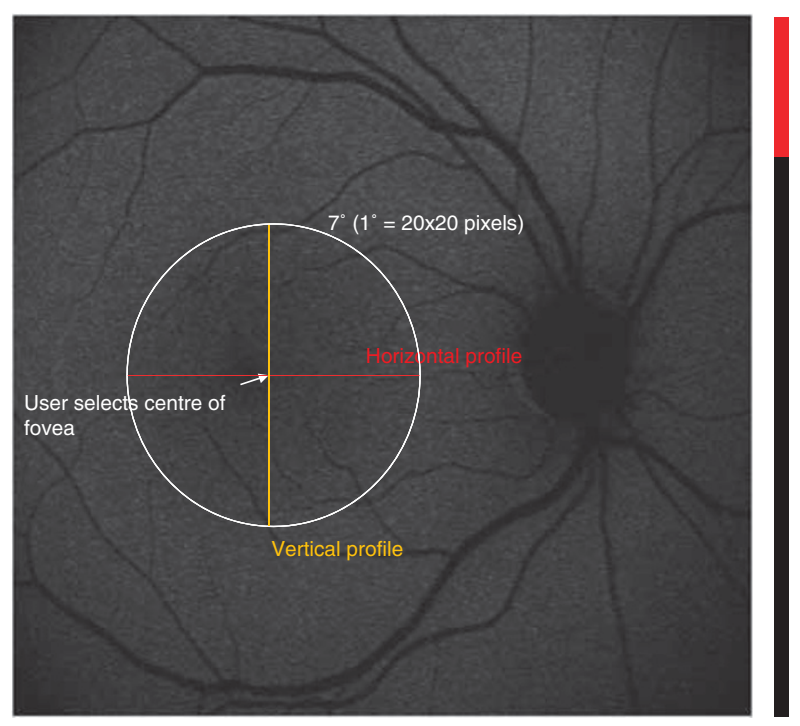

Figure $1 \mathrm{MP}$ density measured in a $7^{\circ}$ radius from the centre of the fovea.

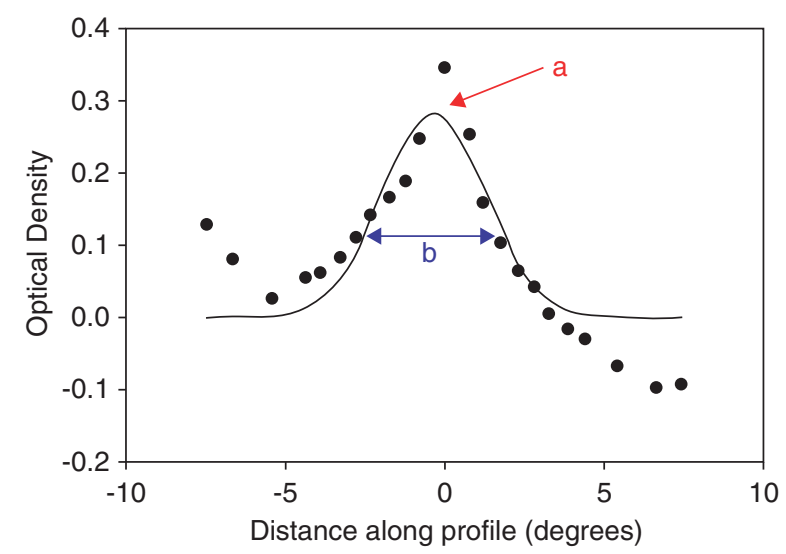

Figure 2 Graph of MP spectral profile (horizontal) explaining ' $a$ ' (rPMPD) and ' $b$ ' (rSDMP) values.

absorbance at 488 and $460 \mathrm{~nm} \cdot{ }^{35}$ Relative MP optical density (MPD) values were calculated for the horizontal and vertical meridians from the FAF images. The values were then exported into Sigmaplot 7.0 (Systat Software, Inc., Richmond, CA, USA) to plot the MP spatial distribution profile horizontally (SDMP). The vertical optical density profile was not plotted as vertical meridians often intersected vascular arcades, causing frequent localized peaks in intensity along the arms of distribution curves that interfered with the results. A curve of best fit was drawn according to the horizontal MP spatial distribution profile and two values were measured: (a) relative PMPD (rPMPD) and (b) relative SDMP (rSDMP), which is represented by the width of the curve at half peak (Figure 2). 


\section{Statistical analysis}

Data analysis and statistical tests were performed using SPSS 16.0 (SPSS Inc., Chicago, IL, USA). Parametric and nonparametric tests were used to compare continuous variables according to data distribution, and relevant graphs were drawn. Relative PMPD and relative SDMP were assessed using $t$-tests and the association between twin pairs was considered using correlation statistics (Pearson product moment). A nonparametric bootstrap procedure written in MatLab (MatLab 7.0.1, MathWorks Inc.), which has been described previously ${ }^{31}$ was used to determine the Pearson product moment correlation coefficients. This enables more robust estimation of parameters in smaller samples. Regression analysis was carried out to investigate the relationship between age relative to $\mathrm{rPMPD}$ and relative to $\mathrm{rSDMP}$.

\section{Results}

\section{Demographics}

A total of 708 twins (354 pairs) participated in the overall AMD twin study; however, only a subset took part in this component as the SLO was located in only one of the data collection clinics. A total of 86 subjects (see Table 1) or 43 twin pairs (21 MZ and $22 \mathrm{DZ}$ ) (27 male, 59 female) aged from 55 to 76 years (mean $62.2 \pm 5.3$ years) had high-quality gradable images ( $\geq$ grade 3 ). Just under half of the sample (37 out of $86,43 \%$ ) presented with features of early AMD (drusen $\geq 63 \mu \mathrm{m}$ or pigmentary irregularities). There were 17 twin pairs in whom both twins had normal fundi, 15 twin pairs where 1 twin had early AMD (drusen and pigmentary change), and 11 twin pairs where both were diagnosed with early AMD. Early AMD concordance rates were $29 \%$ in MZ twin pairs and $23 \%$ in DZ twin pairs. There were no significant differences between $\mathrm{MZ}$ and $\mathrm{DZ}$ pairs based on demographics (Table 1).

\section{Analysis of MP density and distribution}

Measurements of rPMPD (a) and rSDMP (b) in each individual's left and right eyes correlated significantly,

Table 1 Twin demographic characteristics

\begin{tabular}{lccc}
\hline & $\begin{array}{c}\text { MZ } \\
(\mathrm{n}=42, \\
21 \text { pairs })\end{array}$ & $\begin{array}{c}\text { DZ } \\
(\mathrm{n}=44, \\
22 \text { pairs })\end{array}$ & $\begin{array}{c}\text { MZ vs DZ } \\
\left(\mathrm{t} \text {-test or } \chi^{2}\right)\end{array}$ \\
\hline Mean age (SD) & 62.2 & 62.0 & 0.862 \\
Gender (male) & 14 & 13 & 0.817 \\
Early AMD present & 18 & 19 & 1.000 \\
Ever smoked & 9 & 11 & 0.800 \\
Cataract operation & 3 & 5 & 0.330 \\
\hline
\end{tabular}

with an intraclass correlation $(r)$ of $0.86(P<0.001)$ for a and $0.77(P<0.001)$ for $b$. Similar values of rPMPD (right, $0.351 \pm 0.103$; left, $0.358 \pm 0.127$ ) and rSDMP (right, $1.75 \pm 0.398$; left, $1.76 \pm 0.369$ ) were obtained for the right and left eyes and showed no statistical difference. Therefore, in subsequent analysis we only used the right eyes of each individual to avoid introducing error into the analysis of comparisons between twins. Figure 3 shows the typical profiles for a pair of MZ and DZ twins, illustrating the close correlation in $\mathrm{PPMPD}$ in the MZ pair compared with the DZ pair.

An independent samples $t$-test was used to compare rPMPD values. There was a significant difference $(P=0.033$; mean difference $0.043 \pm 0.020)$ between $\mathrm{MZ}$ (mean, $0.373 \pm 0.106$ ) and DZ (mean, $0.330 \pm 0.756$ ) twin pairs. When comparing rPMPD within pairs a significantly higher correlation was found within MZ pairs as compared with that within DZ pairs, represented by Pearson correlations $(r)$ of $0.99,95 \%$ confidence intervals (95\% CI) 0.93 to 1.00$)$ and 0.22 (95\% CI -0.34 to 0.71 ), respectively (Figure 4).

rSDMP was similar for MZ and DZ twin pairs $(P=0.551$; mean difference, $0.047 \pm 0.079)$. rSDMP showed a slightly higher correlation within DZ twin pairs ( $r=0.63$; 95\% CI 0.32 to 0.83$)$ than $\mathrm{MZ}$ twin pairs $(r=0.48 ; 95 \% \mathrm{CI}-0.02$ to 0.83$)$, although this difference was not statistically significant (Figure 4). Thus, rSDMP is unlikely to have a large genetic contribution.

To investigate the relationship of MPD with age, one subject from each twin pair was randomly selected to avoid bias due to their family relationship. Overall, no significant correlation was evident between rPMPD density and age $\left(r^{2}=0.047, P=0.763\right)$ or rSDMP $\left(r^{2}=0.007, P=0.591\right)$. These relationships remained the same when zygosity or AMD status was taken into account.

Using the same form of analysis as for age, no difference between any of the MP parameters was noted when those with normal maculae were compared with those with early AMD (rPMPD 0.36 vs $0.34, t=1.18$ $P=0.243$, rSDMP 1.75 vs $1.75, t=0.028 P=0.977$ ). These relationships remained when zygosity or age were accounted for as well.

\section{Discussion}

This study demonstrates the large contribution genetic factors have in determining PMPD. Our results show that rPMPD values are more highly correlated in MZ twins than DZ twins than those for rSDMP. Our findings on the heritability of PMPD are consistent with those of Liew et al $(\mathrm{MZr}=0.65, \mathrm{DZr}=0.24$, and heritability estimates of 0.67 for heterochromatic flicker photometry whereas $\mathrm{MZr}=0.83, \mathrm{DZr}=0.50$, and heritability estimates of 

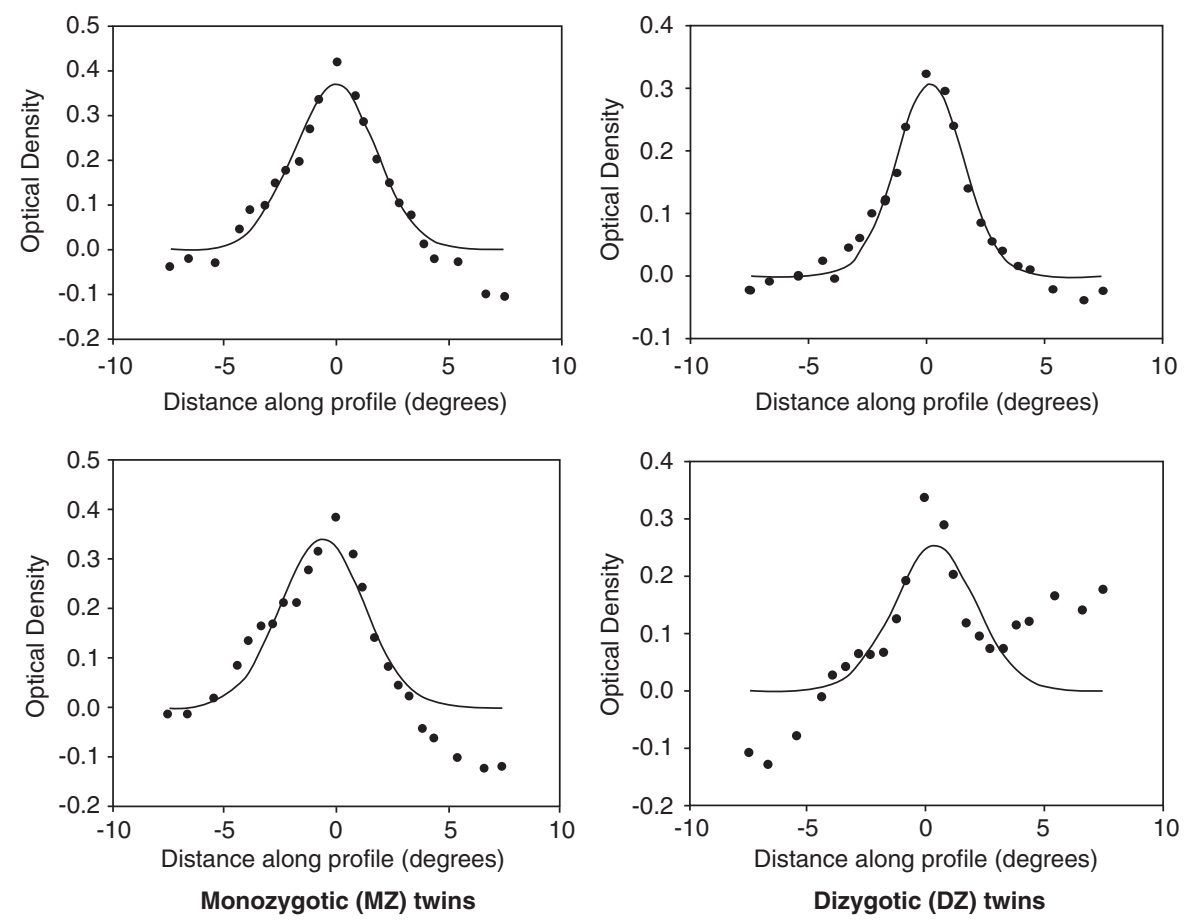

Figure 3 Typical MP spectral profiles for MZ (left) and DZ (right) twins. A closer correlation is observed in the profiles of MZ twins than DZ twins. Twin 1 upper, twin 2 lower.
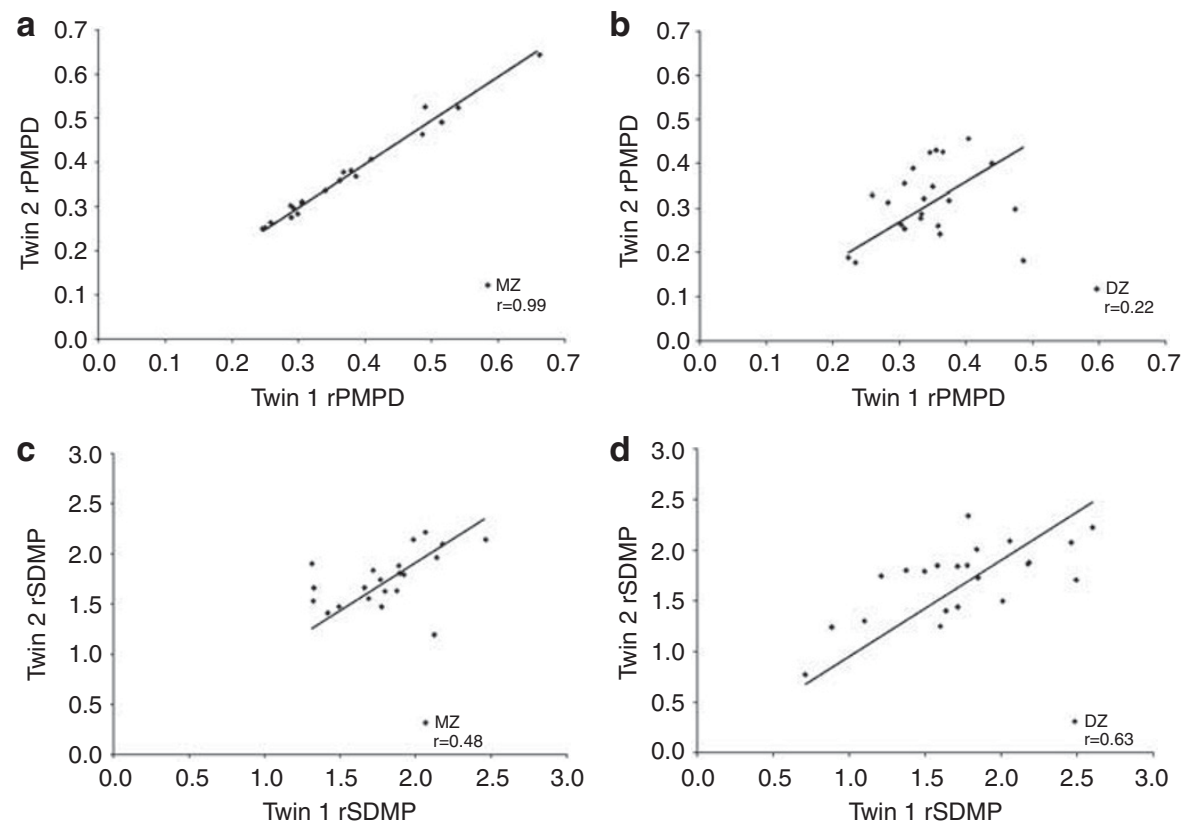

Figure 4 Graphs showing the correlation for rPMPD density between MZ twin pairs (a) and DZ twin pairs (b) and the correlation for the relative rSDMP between MZ twin pairs (c) and DZ twin pairs (d).

0.85 for FAF images). ${ }^{21}$ Although substantial heritability has been reported by other studies focusing at anatomical ocular structures such as macular thickness, ${ }^{32}$ optic nerve head biometry, ${ }^{36}$ and cup-to-disc ratio, ${ }^{37}$ the striking concordance noted between MZ twin pairs for rPMPD (Figure 4) is somewhat surprising, given the 
large emphasis placed on environmental factors such as diet and supplementation in the general MP literature. Taken with the previous reports of heritability in excess of $70 \%$, these findings suggest that genetic factors exert an overwhelming influence on the amount of MP that is deposited in the eye. Given that several specific

xanthrophyll-binding proteins have been identified, ${ }^{38,39}$ including the Pi isoform of gluthathione S-transferase, ${ }^{38}$ it is possible that specific polymorphisms may exist that influence the uptake, metabolism or deposition of $\mathrm{L}$ and $Z$. Indeed, genetic factors are likely to account for part of the variance unaccounted for in studies investigating the relationships between dietary, serum, and macular carotenoid concentrations.

We did not find evidence to suggest that the SDMP was genetically determined, implying that the distribution of MP might be more influenced by other lifestyle risk factors, and thus possibly more able to be manipulated though diet. The high degree of intersubject variability in spatial distribution is also consistent with this interpretation. This is the first study to evaluate the heritability of the spatial profile. Liew et $a l^{21}$ reported that $\mathrm{MZ}$ profiles tended to look more alike than DZ profiles, suggesting a possible genetic influence, but did not subject it to formal analysis. A recent population-based study that looked specifically at the spatial profile of MP using dual-wavelength analysis of autofluorescence images found that a ring-like structure of MP was present in almost $20 \%$ of the participants, tended to be bilateral and was significantly more common in females and never smokers. ${ }^{40}$

Although substantial progress has been made in understanding mechanisms involved in the uptake, metabolism, and deposition of $\mathrm{L}$ and $\mathrm{Z},{ }^{39,41}$ it is clearly a complex process. The ratio of $\mathrm{L} / \mathrm{Z} /$ meso-zeaxanthin varies with retinal profile with $Z$ predominant at the central peak and $L$ predominant in the peripheral retina. Meso-zeaxanthin, a stereoisomer of $\mathrm{Z}$ not normally present in diet or serum reaches its peak at the point in the retina where $L / Z$ ratio is at a minimum and is considered a metabolic product of L. ${ }^{42}$ These are all known to differ in terms of antioxidant capacity, their localization within cells and the binding proteins associated with their deposition. It is not unreasonable to imagine that the impact of processes that either augment or deplete their concentration could vary with eccentricity as the ratio of $\mathrm{L} / \mathrm{Z} / \mathrm{Meso}-\mathrm{Z}$ changes. Indeed, several studies focusing at the spatial profile of MP in response to supplementation have shown that $\mathrm{MP}$ is not augmented equally across the retina ${ }^{43}$ and appears to be deposited more in peripheral locations, demonstrating that SDMP can be altered by environmental manipulation which is consistent with our findings. However, the potential impact on macular health of being able to manipulate the SDMP is not at all clear.

In the analysis of the age relationship with MP we were unable to find any significant association between age and both optical density and spatial distribution. The results of previous studies investigating this relationship have also been inconsistent. $7,9,44,45$

When $\mathrm{PPMPD}$ and rSDMP were compared in those with early AMD and those without, we did not find any significant difference, which is in keeping with the literature to date as with the exception of a few studies, ${ }^{6-8}$ most studies comparing those with drusen and those without have been unable to find a cross-sectional association, ${ }^{9-11}$ including the large carotenoids in Age-Related Eye Disease Study $(n=1698) .{ }^{10}$

Our study also demonstrated the ability to rapidly measure both MP optical density and spatial distribution from a single AF image; however, there are some difficulties present with regard to measuring MP from FAF images using the one-wavelength method. The technique is based on the assumption that the quantity of FAF in the absence of MP has a uniform distribution across the field, which is not the case in vivo. Thus, heterogeneous redistributions of lipofuscin fluorophores could be misinterpreted as absolute changes in MP distribution. Additionally, previous studies utilizing this method $^{34,46}$ have reported that on the basis of different absorption characteristics of other fluorophores such as melanin in the RPE and haemoglobin, the amount and allocation of MP could be overestimated. Areas of low MP density are especially prone. ${ }^{47}$ However, all our images were subject to the same limitations and it should not detract from our overall conclusions about trends in hereditability of MP in MZ and DZ twins. The strength inherent of a classic twin study rests on the utility of reproducible techniques for all subjects and the conclusions are determined by the relative nature of the readings rather than absolute levels.

The small number of twin pairs resulting from the initial triage of participation owing to the location of the machine should not have introduced bias. The small numbers not only limited the power of our study but prevented us from undertaking structural equation modelling to ascertain full heritability. However, the bootstrapping procedure, which we also used in a previous study, ${ }^{31}$ enables robust parameter estimates to be calculated from small samples ${ }^{48}$ and makes no prior assumptions as to the distribution of the population. ${ }^{48,49}$

Additionally, although best efforts were made to match image quality by image processing, other factors such as media opacities (eg, cataracts) that decrease the FAF image contrast and the uneven distribution of fundus 
illumination may affect readings of the mean SE, which compares the fundamental differences between two images. However, our mean MP optical density of $0.35 \pm 0.096$ using our one-wavelength autofluorescence method, which measured MP optical density at $1 \%$ eccentricity from the foveal centre, correlates well with that of Robson et al ${ }^{47}(0.37 \pm 0.11)$, who also used FAF imaging and an identical MP density calculation programme, though slightly lower than those reported by the previous twin study that also used auto fluorescence $(0.27 \pm 0.10) .^{21}$ Twin subjects' values for PMPD and spatial distribution covered a wide range, which is consistent with many studies that have reported considerable interindividual variability in both variables.

Within the overall twin study all participants underwent slit lamp evaluation at examination and cataracts were graded using the Wilmer system of grading lens opacities and each participant was given a nuclear opacity grading score ranging from 1 to 4 , with 4 being the greatest opacity. ${ }^{50}$ Although a recent study reported the potential impact of nuclear cataract on MP measurements using autofluorescence, ${ }^{51}$ we did not attempt adjust for lens status in our analysis as all of the participants had a central cataract grading of either 1 or 2 with no difference in the concordance of grades between MZ and DZ (MZ and DZ) (data not shown). Given the low levels of cataract present and the fact that any twin pair would not differ by more than one grade, it is unlikely that lens status could account for the findings in this study.

In this same cohort of twins we have also reported on the hereditability of macular thickness ${ }^{32}$ as well as performance on several psychophysical retinal visual function tests. ${ }^{31}$ We found that although the ability to see colour and flicker thresholds were strongly inherited, the dark adaptation curve was not. We interpreted these findings to imply that changes in Bruch's membrane, which are thought to dictate the flux of vitamin A derivatives to the RPE and photoreceptors, and thus impact on dark adaptation, are also not particularly influenced by genes and thus potentially able to be altered through environmental changes. Thus, we have found that both Bruch's membrane changes and the distribution of macular pigment are not strongly influenced by genetic differences even though the disease itself is considered highly influenced by genetic polymorphisms in several well-characterized genes. ${ }^{52-54}$ Thus, these two parameters, which are potentially very important influences on the development of AMD may be able to be influenced through lifestyle choices, and thus amenable to therapeutic intervention such as dietary changes and oral supplementation.

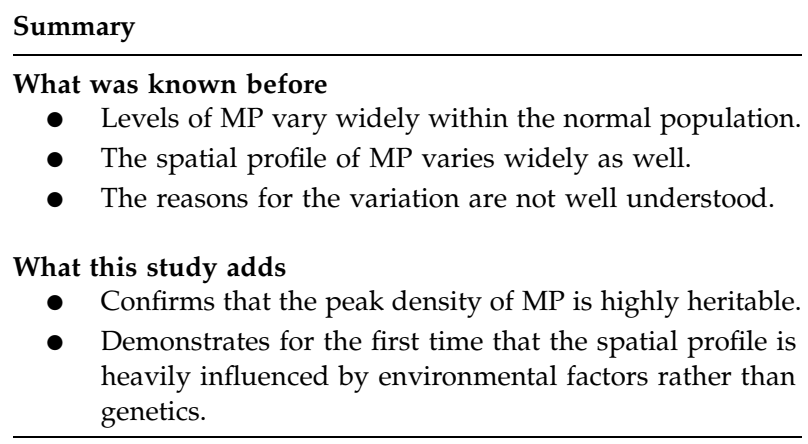

\section{Conflict of interest}

The authors declare no conflict of interest.

\section{Acknowledgements}

We thank Dr Andrew Anderson for his help in writing the software used for the bootstrapping procedure. We thank the Australian Twin Registry for access to this national resource. This work was supported by the National Health and Medical Research Council (NHMRC) Centre for Clinical Research Excellence (\#529923), NHMRC Career Development Award (RHG), and a NHMRC practitioner fellowship (RHG). This work was also supported by a grant from the Ophthalmic Research Institute of Australia. CERA receives Operational Infrastructure Support from the Victorian Government.

\section{References}

1 Khachik F, Englert G, Daitch CE, Beecher GR, Tonucci LH, Lusby WR. Isolation and structural elucidation of the geometrical isomers of lutein and zeaxanthin in extracts from human plasma. J Chromatogr 1992; 582(1-2): 153-166.

2 Landrum JT, Bone RA. Lutein, zeaxanthin, and the macular pigment. Arch Biochem Biophys 2001; 385(1): 28-40.

3 Junghans A, Sies H, Stahl W. Macular pigments lutein and zeaxanthin as blue light filters studied in liposomes. Arch Biochem Biophy 2001; 391: 160-164.

4 Algvere PV, Marshall J, Seregard S. Age-related maculopathy and the impact of blue light hazard. Acta Ophthalmologica Scandinavica 2006; 84(1): 4-15.

5 Krinsky NI, Landrum JT, Bone RA. Biologic mechanisms of the protective role of lutein and zeaxanthin in the eye. Annu Rev Nutr 2003; 23: 171-201.

6 Bernstein PS, Zhao DY, Wintch SW, Ermakov IV, McClane RW, Gellermann W. Resonance Raman measurement of macular carotenoids in normal subjects and in age-related macular degeneration patients. Ophthalmology 2002; 109(10): 1780-1787.

7 Beatty S, Murray IJ, Henson DB, Carden D, Koh H, Boulton ME. Macular pigment and risk for age-related macular degeneration in subjects from a northern European population. Invest Ophthalmol Vis Sci 2001; 42(2): 439-446. 
8 Wustemeyer H, Jahn C, Nestler A, Barth T, Wolf S. A new instrument for the quantification of macular pigment density: first results in patients with AMD and healthy subjects. Graefes Arch Clin Exp Ophthalmol 2002; 240(8): 666-671.

9 Jahn C, Wustemeyer H, Brinkmann C, Trautmann S, Mossner A, Wolf S. Macular pigment density in age-related maculopathy. Graefes Arch Clin Exp Ophthalmol 2005; 243(3): 222-227.

10 LaRowe TL, Mares JA, Snodderly DM, Klein ML, Wooten BR, Chappell R. Macular pigment density and age-related maculopathy in the carotenoids in age-related eye disease study. An ancillary study of the women's health initiative. Ophthalmology 2008; 115(5): 876-883; e871.

11 Berendschot TT, Willemse-Assink JJ, Bastiaanse M, de Jong PT, van Norren D. Macular pigment and melanin in age-related maculopathy in a general population. Invest Ophthalmol Vis Sci 2002; 43(6): 1928-1932.

12 Bone RA, Landrum JT, Mayne ST, Gomez CM, Tibor SE, Twaroska EE. Macular pigment in donor eyes with and without AMD: a case-control study. Invest Ophthalmol Vis Sci 2001; 42(1): 235-240.

13 Ciulla TA, Curran-Celantano J, Cooper DA, Hammond Jr BR, Danis RP, Pratt LM et al. Macular pigment optical density in a midwestern sample. Ophthalmology 2001; 108(4): 730-737.

14 Hammond Jr BR, Caruso-Avery M. Macular pigment optical density in a Southwestern sample. Invest Ophthalmol Vis Sci 2000; 41(6): 1492-1497.

15 Hammond Jr BR, Curran-Celentano J, Judd S, Fuld K, Krinsky NI, Wooten BR et al. Sex differences in macular pigment optical density: relation to plasma carotenoid concentrations and dietary patterns. Vision Res 1996; 36(13): 2001-2012.

16 Hammond Jr BR, Wooten BR, Snodderly DM. Cigarette smoking and retinal carotenoids: implications for agerelated macular degeneration. Vision Res 1996; 36(18): 3003-3009.

17 Ciulla TA, Hammond Jr BR. Macular pigment density and aging, assessed in the normal elderly and those with cataracts and age-related macular degeneration. Am J Ophthalmol 2004; 138(4): 582-587.

18 Curran-Celentano J, Hammond Jr BR, Ciulla TA, Cooper DA, Pratt LM, Danis RB. Relation between dietary intake, serum concentrations, and retinal concentrations of lutein and zeaxanthin in adults in a Midwest population. Am J Clin Nutr 2001; 74(6): 796-802.

19 Nolan J, O'Donovan O, Kavanagh H, Stack J, Harrison M, Muldoon A et al. Macular pigment and percentage of body fat. Invest Ophthalmol Vis Sci 2004; 45(11): 3940-3950.

20 Hammond Jr BR, Johnson EJ, Russell RM, Krinsky NI, Yeum KJ, Edwards RB et al. Dietary modification of human macular pigment density. Invest Ophthalmol Vis Sci 1997; 38(9): 1795-1801.

21 Liew SH, Gilbert CE, Spector TD, Mellerio J, Marshall J, van Kuijk FJ et al. Heritability of macular pigment: a twin study. Invest Ophthalmol Vis Sci 2005; 46(12): 4430-4436.

22 Hammond Jr BR, Wooten BR, Snodderly DM. Individual variations in the spatial profile of human macular pigment. J Opt Soc Am A Opt Image Sci Vis 1997; 14(6): 1187-1196.

23 Sharifzadeh M, Zhao DY, Bernstein PS, Gellermann W. Resonance Raman imaging of macular pigment distributions in the human retina. J Opt Soc Am A Opt Image Sci Vis 2008; 25(4): 947-957.
24 Bone RA, Landrum JT, Fernandez L, Tarsis SL. Analysis of the macular pigment by HPLC: retinal distribution and age study. Invest Ophthalmol Vis Sci 1988; 29(6): 843-849.

25 Bone RA, Landrum JT, Friedes LM, Gomez CM, Kilburn MD, Menendez E et al. Distribution of lutein and zeaxanthin stereoisomers in the human retina. Exp Eye Res 1997; 64(2): 211-218.

26 Snodderly DM, Auran JD, Delori FC. The macular pigment. II. Spatial distribution in primate retinas. Invest Ophthalmol Vis Sci 1984; 25(6): 674-685.

27 Trieschmann M, Spital G, Lommatzsch A, van Kuijk E, Fitzke F, Bird AC et al. Macular pigment: quantitative analysis on autofluorescence images. Graefes Arch Clin Exp Ophthalmol 2003; 241(12): 1006-1012.

28 Berendschot TT, van Norren D. Macular pigment shows ringlike structures. Invest Ophthalmol Vis Sci 2006; 47(2): 709-714.

29 Nolan JM, Stringham JM, Beatty S, Snodderly DM. Spatial profile of macular pigment and its relationship to foveal architecture. Invest Ophthalmol Vis Sci 2008; 49(5): 2134-2142.

30 Kirby ML, Galea M, Loane E, Stack J, Beatty S, Nolan JM. Foveal anatomic associations with the secondary peak and the slope of the macular pigment spatial profile. Invest Ophthalmol Vis Sci 2009; 50(3): 1383-1391.

31 Hogg RE, Dimitrov PN, Dirani M, Varsamidis M, Chamberlain MD, Baird PN et al. Gene-environment interactions and aging visual function: a classical twin study. Ophthalmology 2009; 116(2): 263-269.

32 Chamberlain MD, Guymer RH, Dirani M, Hopper JL, Baird PN. Heritability of macular thickness determined by optical coherence tomography. Invest Ophthalmol Vis Sci 2006; 47(1): 336-340.

33 Martin NG, Martin PG. The inheritance of scholastric abilities in a sample of twins. I. Ascertainments of the sample and diagnosis of zygosity. Ann Hum Genet 1975; 39(2): 213-218.

34 Trieschmann M, Heimes B, Hense HW, Pauleikhoff D. Macular pigment optical density measurement in autofluorescence imaging: comparison of one- and two-wavelength methods. Graefes Arch Clin Exp Ophthalmol 2006; 244(12): 1565-1574.

35 Stockman A, Sharpe LT, Fach C. The spectral sensitivity of the human short-wavelength sensitive cones derived from thresholds and color matches. Vis Res 1999; 39(17): 2901-2927.

36 Hewitt AW, Poulsen JP, Alward WL, Bennett SL, Budde WM, Cooper RL et al. Heritable features of the optic disc: a novel twin method for determining genetic significance. Invest Ophthalmol Vis Sci 2007; 48(6): 2469-2475.

37 Teikari JM, Airaksinen JP. Twin study on cup/disc ratio of the optic nerve head. Br J Ophthalmol 1992; 76(4): 218-220.

38 Bhosale P, Larson AJ, Frederick JM, Southwick K, Thulin $\mathrm{CD}$, Bernstein PS. Identification and characterization of a $\mathrm{Pi}$ isoform of glutathione $S$-transferase (GSTP1) as a zeaxanthin-binding protein in the macula of the human eye. J Biol Chem 2004; 279(47): 49447-49454.

39 Bhosale P, Li B, Sharifzadeh M, Gellermann W, Frederick JM, Tsuchida $\mathrm{K}$ et al. Purification and partial characterization of a lutein-binding protein from human retina. Biochemistry 2009; 48(22): 4798-4807.

40 Dietzel M, Zeimer M, Heimes B, Pauleikhoff D, Hense HW. The ringlike structure of macular pigment in age-related maculopathy: results from the muenster aging and retina 
study (Mars). Invest Ophthalmol Vis Sci 2011; 52(11): 8016-8024

41 Loane E, Nolan JM, O'Donovan O, Bhosale P, Bernstein PS, Beatty S. Transport and retinal capture of lutein and zeaxanthin with reference to age-related macular degeneration. Surv Ophthalmol 2008; 53(1): 68-81.

42 Bone RA, Landrum JT. Distribution of macular pigment components, zeaxanthin and lutein, in human retina. Methods Enzymol 1992; 213: 360-366.

43 Wenzel AJ, Sheehan JP, Gerweck C, Stringham JM, Fuld K, Curran-Celentano J. Macular pigment optical density at four retinal loci during 120 days of lutein supplementation. Ophthalmic Physiol Opt 2007; 27(4): 329-335.

44 Beatty S, Boulton M, Henson D, Koh HH, Murray IJ. Macular pigment and age related degeneration. $\mathrm{Br} J$ Ophthalmol 1999; 83(7): 867-877.

45 Berendschot TT, van Norren D. On the age dependency of the macular pigment optical density. Exp Eye Res 2005; 81(5): 602-609.

46 Robson AG, Moreland JD, Pauleikhoff D, Morrissey T, Holder GE, Fitzke FW et al. Macular pigment density and distribution: comparison of fundus autofluorescence with minimum motion photometry. Vis Res 2003; 43(16): 1765-1775.

47 Robson AG, Harding G, van Kuijk FJ, Pauleikhoff D, Holder $\mathrm{GE}$, Bird AC et al. Comparison of fundus autofluorescence and minimum-motion measurements of macular pigment distribution profiles derived from identical retinal areas. Perception 2005; 34(8): 1029-1034.
48 Foster DH, Bischof WF. Bootstrap variance estimators for the parameters of small-sample sensory-performance functions. Biol Cybern 1987; 57(4-5): 341-347.

49 Maloney LT. Confidence intervals for the parameters of psychometric functions. Percept Psychophys 1990; 47(2): 127-134.

50 Taylor HR, West SK. A simple system for the clinical grading of lens opacities. Yan Ke Xue Bao 1988; 4(1): 14-18.

51 Sasamoto Y, Gomi F, Sawa M, Sakaguchi H, Tsujikawa M, Nishida K. Effect of cataract in evaluation of macular pigment optical density by autofluorescence spectrometry. Invest Ophthalmol Vis Sci 2011; 52(2): 927-932.

52 Hageman GS, Anderson DH, Johnson LV, Hancox LS, Taiber AJ, Hardisty LI et al. A common haplotype in the complement regulatory gene factor $\mathrm{H}(\mathrm{HF} 1 / \mathrm{CFH})$ predisposes individuals to age-related macular degeneration. Proc Natl Acad Sci USA 2005; 102(20): 7227-7232.

53 McKay GJ, Silvestri G, Patterson CC, Hogg RE, Chakravarthy U, Hughes AE. Further assessment of the complement component 2 and factor $\mathrm{B}$ region associated with age-related macular degeneration. Invest Ophthalmol Vis Sci 2009; 50(2): 533-539.

54 Hughes AE, Orr N, Esfandiary H, Diaz-Torres M, Goodship T, Chakravarthy U. A common $\mathrm{CFH}$ haplotype, with deletion of CFHR1 and CFHR3, is associated with lower risk of age-related macular degeneration. Nat Genet 2006; 38(10): 1173-1177. 